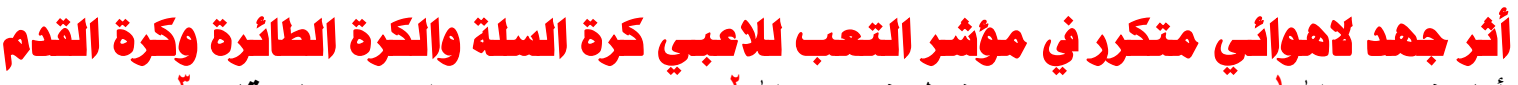

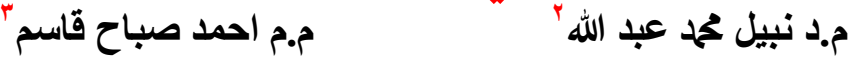 أ.بد أياد محمد عبد الله

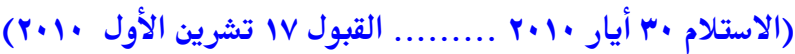

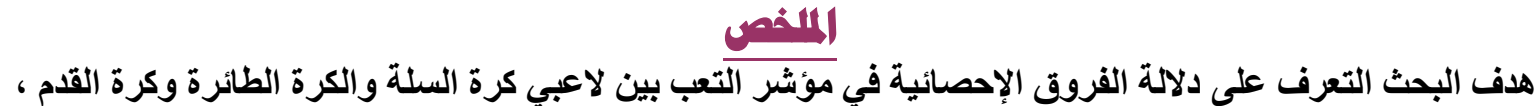

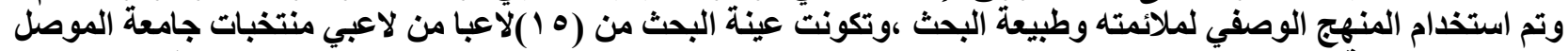

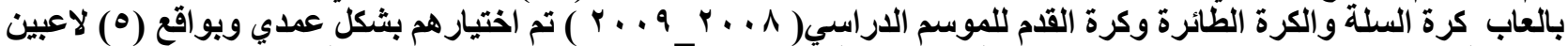

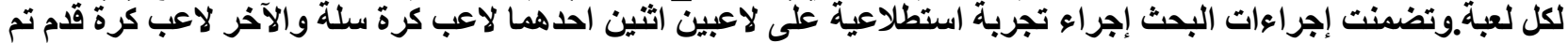

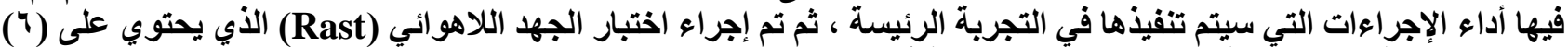

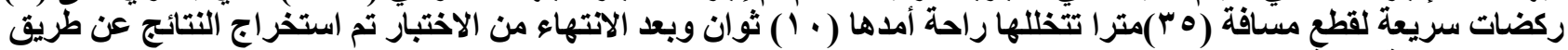

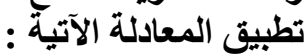

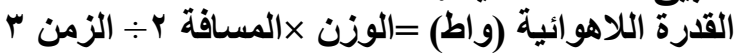

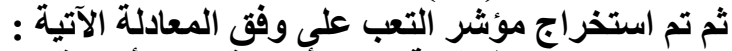

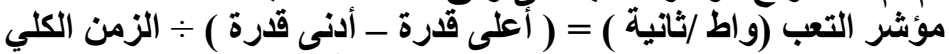

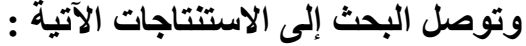

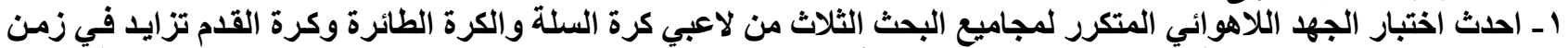

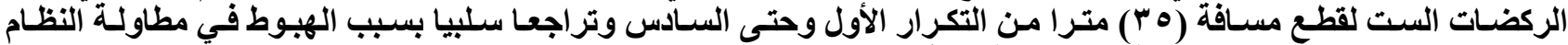

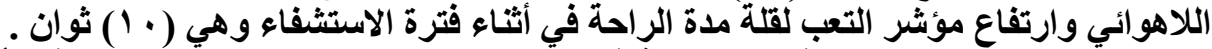

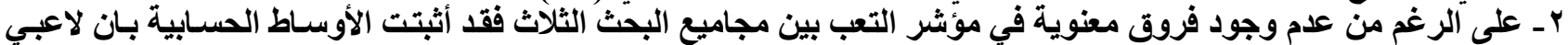

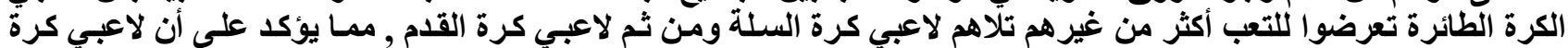

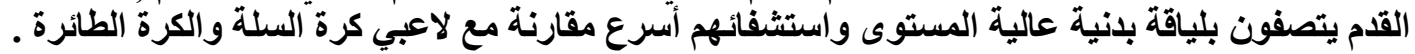

\section{The Impact of Repeated Anaerobic Effort in The Anaerobic Capability and the Fatigue Indicator of Basketball, Volleyball and SOCCER Players}

Prof.Dr.Ayad.M.Abdullah

Lecturer.Dr. Nabeel.M.Abdullah

Assist.Lecturer.Ahmed.S.Qasim

\section{$\underline{\text { Abstract }}$}

The current study aimed at identifying the statistical significances the fatigue indicator among basketball, volleyball and soccer players. The descriptive approach was applied for its appropriateness to the nature of the study. The sample of the study consisted of (15) players representing Mosul University teams of these games for the year (2008-2009); WHOM they were chosen intentionally, (5) players for each game.

The procedures of the research included a pilot study applied on two players; the first a basketball players and the second is a soccer player. Then the anaerobic test of RAST was applied, which consisted of (6)sprint runs passing a distance of (35) meters intervened by rest periods lasted for (10) seconds for each. After ending the test, the results were concluded through applying the following equation:

anaerobic power $($ watt $)=$ weight $x$ distance $^{2} /$ time $^{3}$

The fatigue indicator was concluded by applying the following equation:

$$
\text { '"فرع الالعاب الفردية/كلية التربية الرياضية/جامعة الموصل/العراق. }
$$


The fatigue indicator $(\mathrm{watt} / \mathrm{sec})=$ the highest power- lowest power $) /$ total time.

The research reached at the following:

1. The repeated anaerobic effort of the three groups of basketball, volleyball and soccer players made an increase in the time of the (6) runs that passed (35) meters distance starting from the first run until the sixth one, and that is because of decline in the duration of anaerobic system and the rise of fatigue indicator resulted from short rest time during recovery period that lasts for (10) seconds.

2. Though there are no significant differences in fatigue indicator among the three groups; the means proved "that the teams were matter of fatigue in this order" volleyball players followed by basketball players then came soccer players, the matter which emphasizes that soccer players are characterized with high level of physical fitness and phosphegeneic lactic aerobic power and their recovery is faster in comparison with basketball and volleyball players.

\section{ا - التعريف بالبحث:}

تعد الألعاب الفرقية ومنها العاب كرة السلة وكرة القدم والكرة الطائرة من الالعاب التي تتميز بان اغلب الحركات

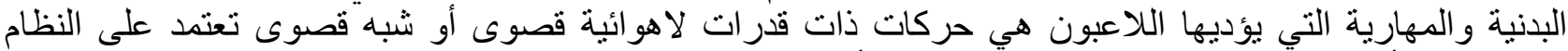

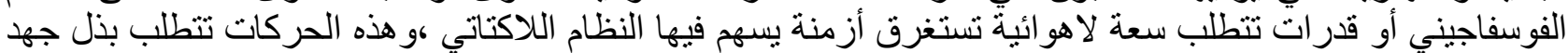

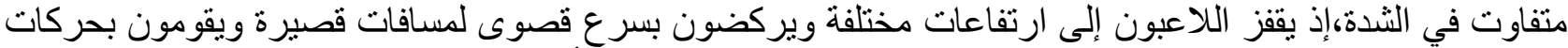

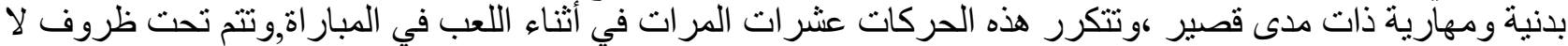

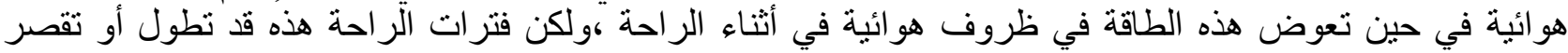

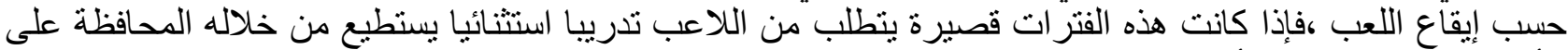

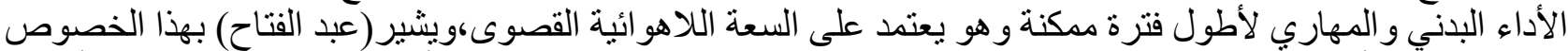

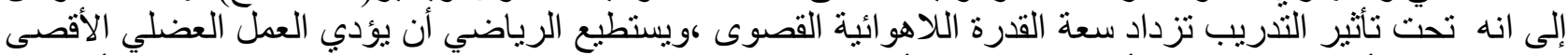

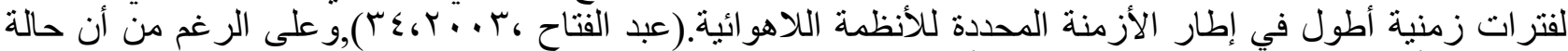

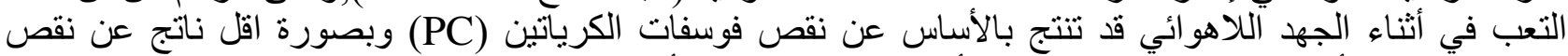

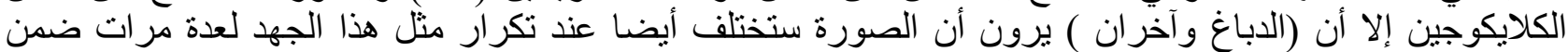

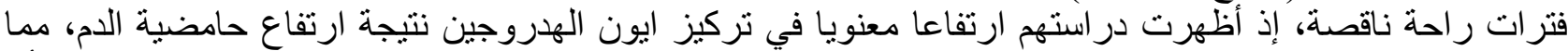

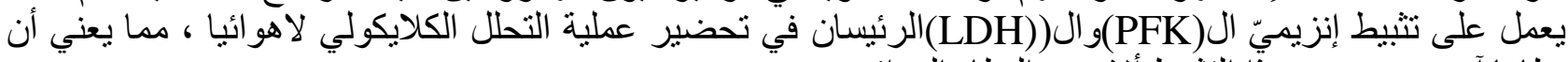

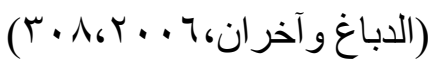

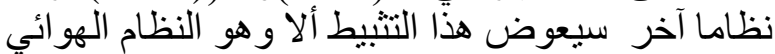

وبناء على ما تقدم وللقناعة التامة بأهمية تدريبات الجهد اللاهو ائي المتكرر في العاب كرة السلة وكرة القدام

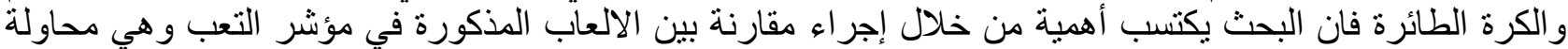

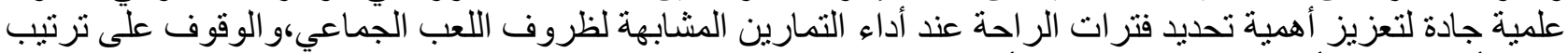

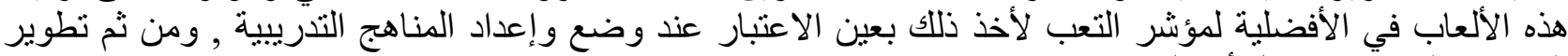

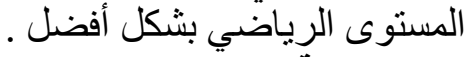

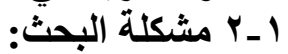

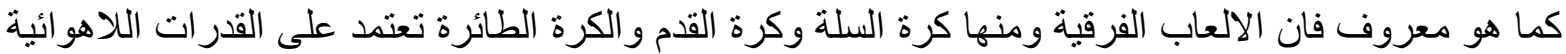

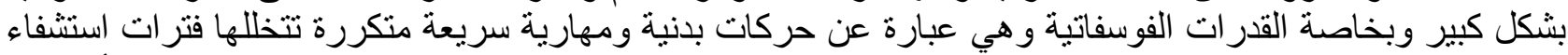

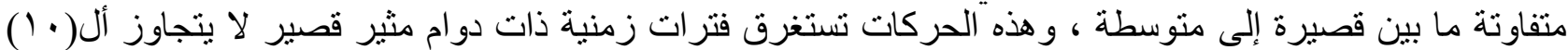

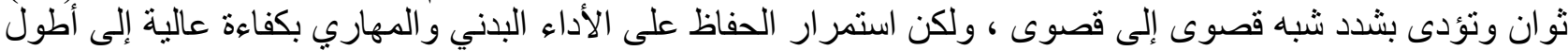

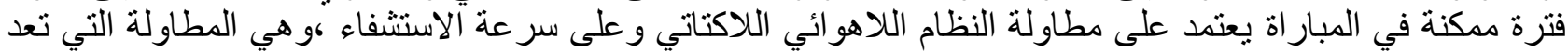

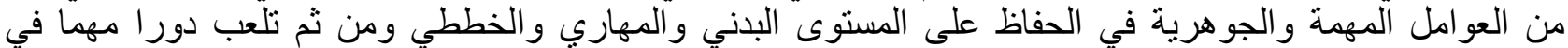

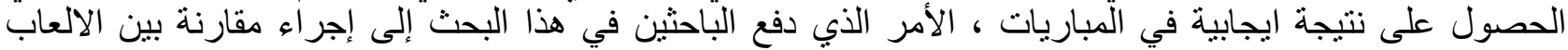

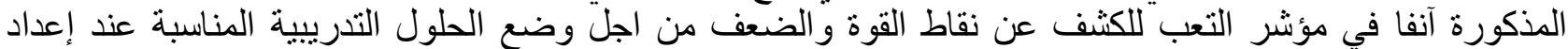

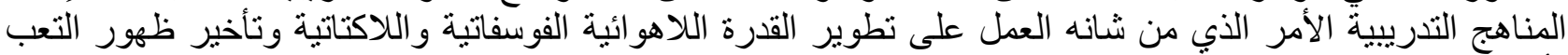


التعرف على دلالة الفروق الإحصائية في مؤشر التعب بين لاعبي لاعبي كرة السلة وكرة القدم و الكرة الطائرة .

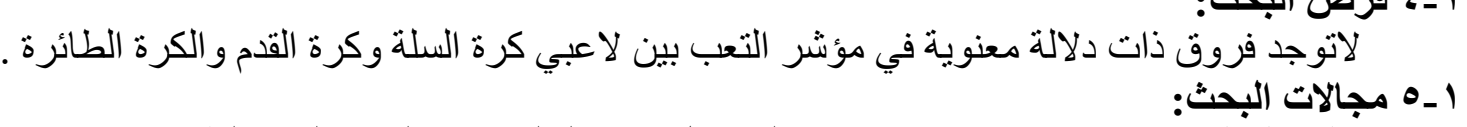

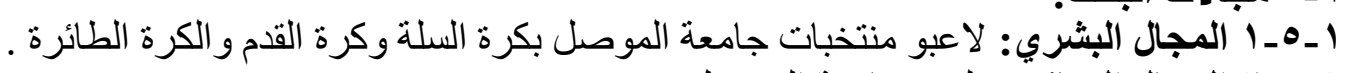

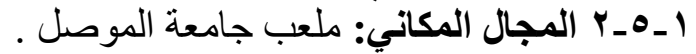

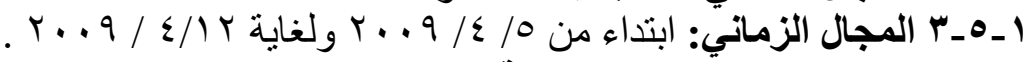

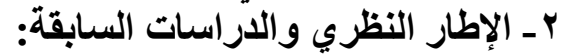

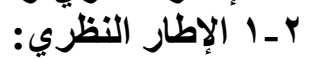

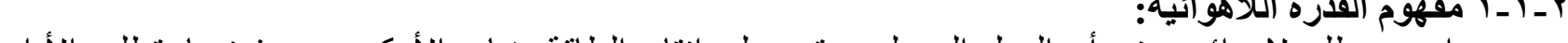

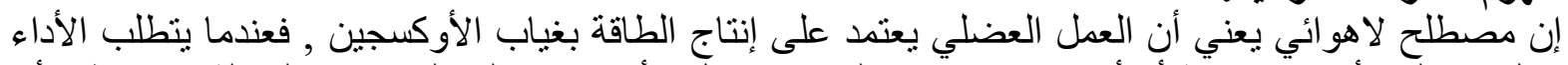

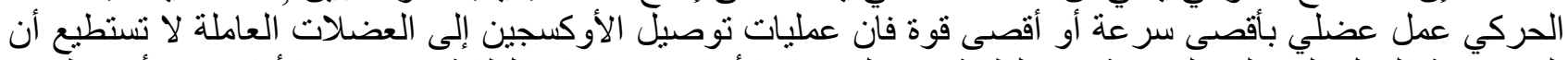

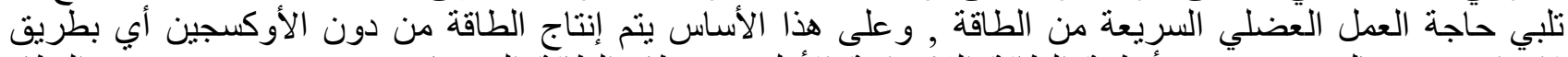

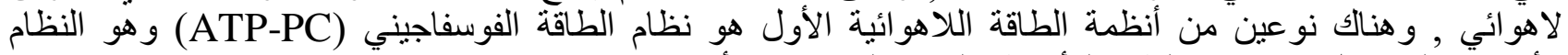

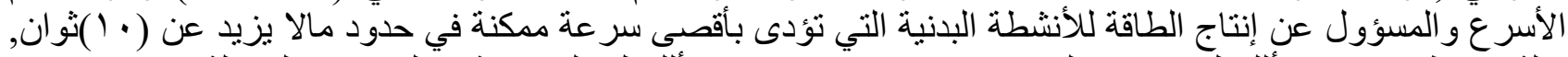

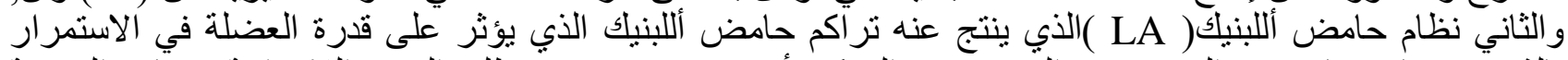

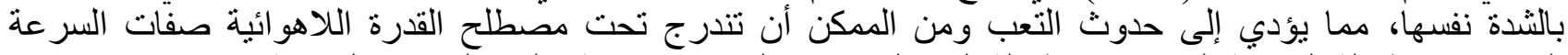

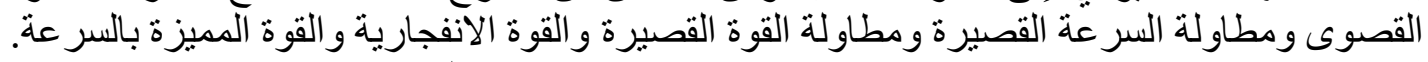

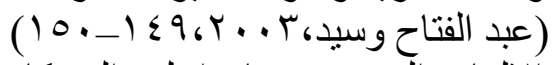

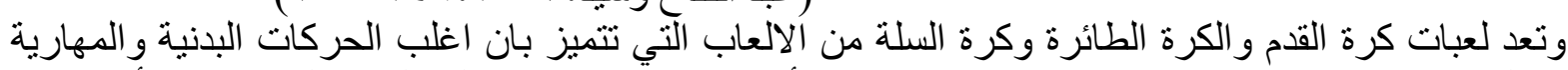

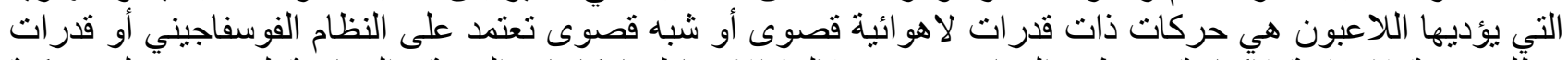

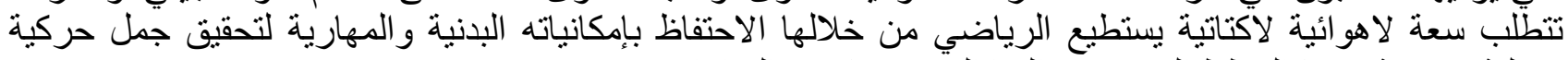

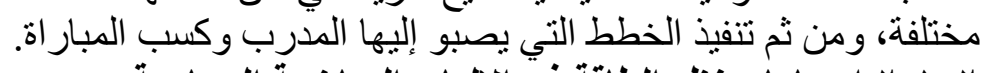

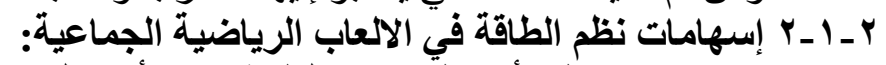

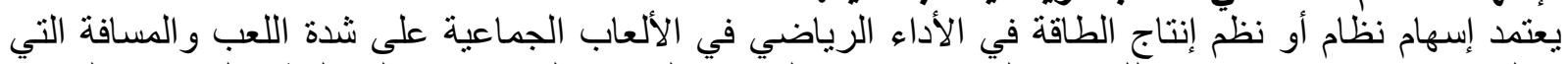

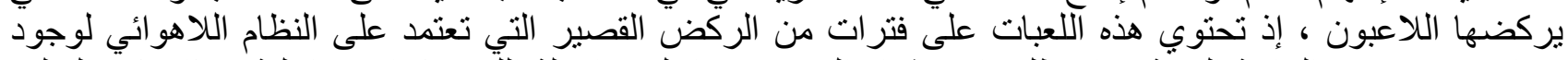

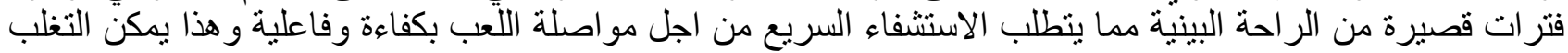

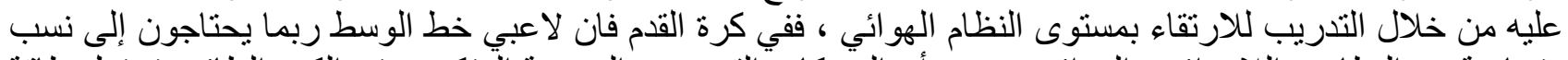

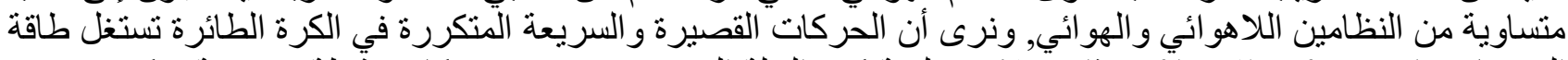

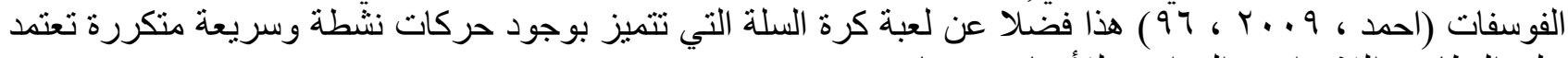

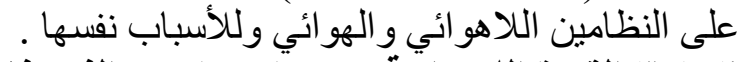

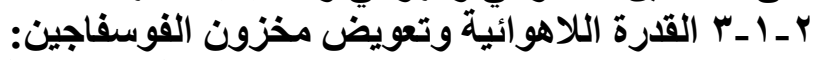

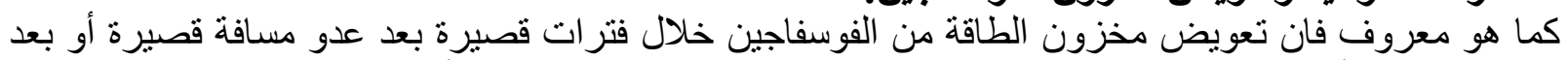

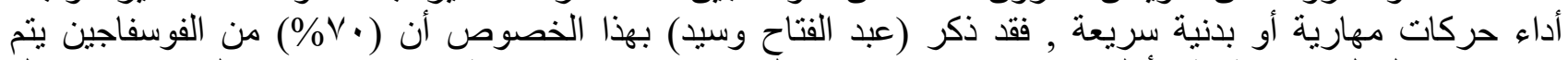

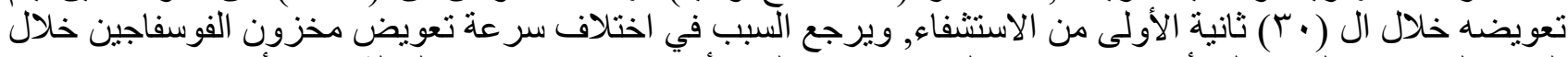

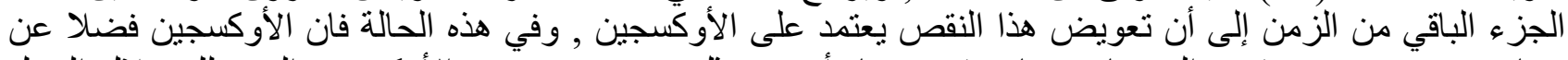

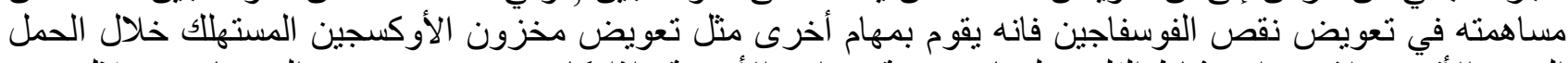

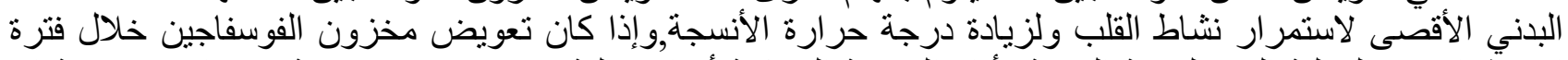

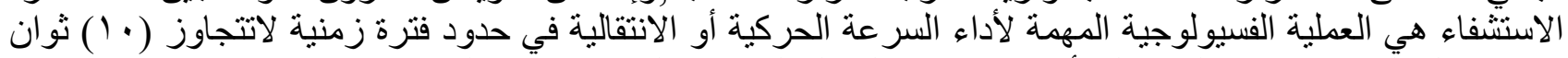

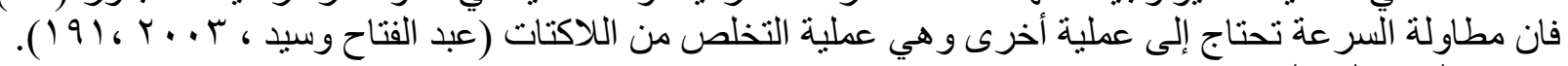

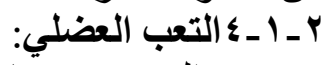
التعب هو عبارة عن هبوطو وقتي في المقدرة على الاستمرار في أداء العمل ، ومواضع التعب تكمن في الجهاز

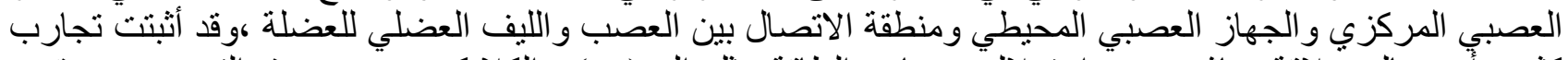

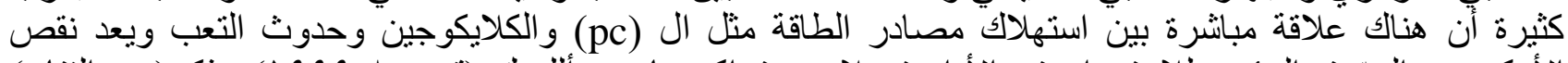

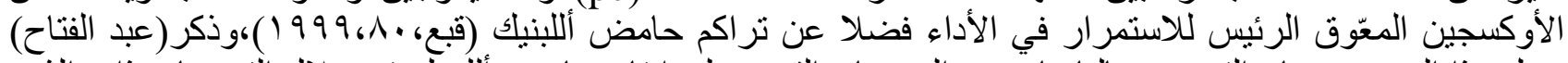

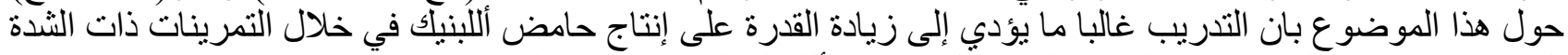

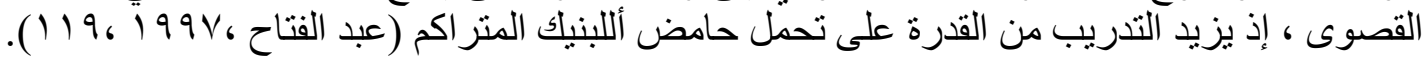




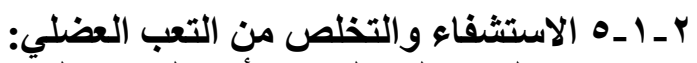

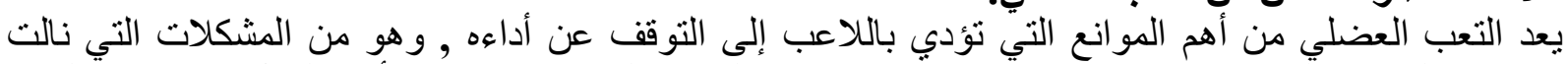

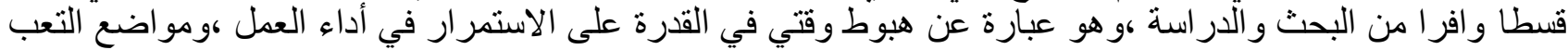

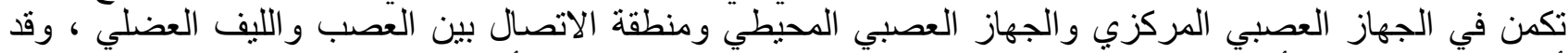

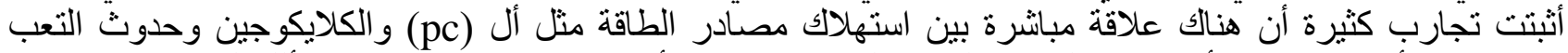
،ويعد نقص أو عدم توفر الأوكسجين المعّوق الرئيس للاستمر ار في الأداء فضلاعن تر اكم حامض أللبنيك.

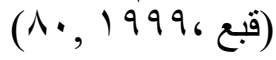

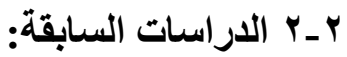

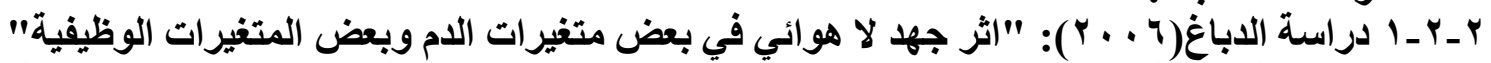

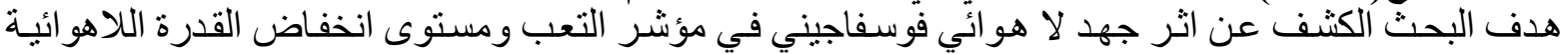

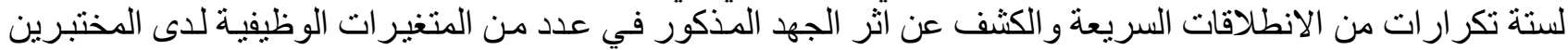

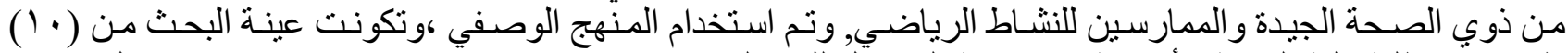

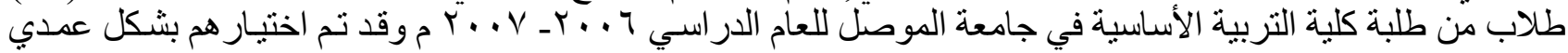

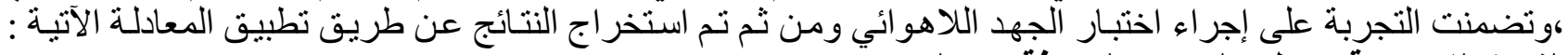

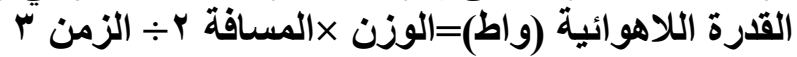

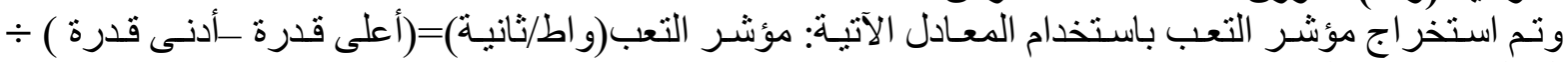

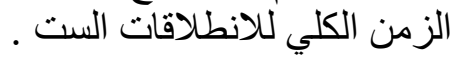

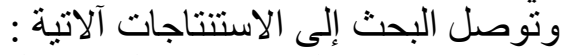

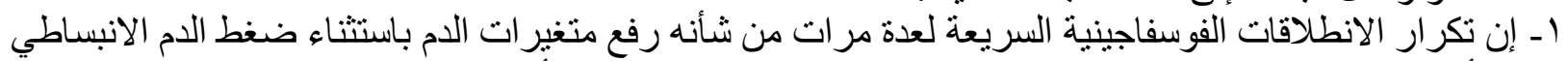

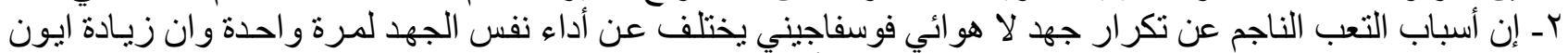

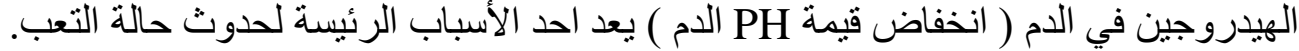

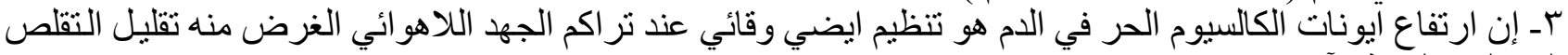

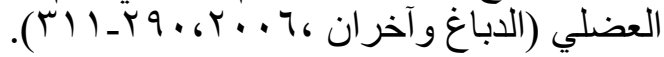

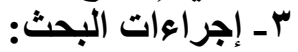

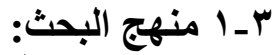
تم استخذام المنهج الوصن: الوصفي لأنه انسب المناهج للوصول إلى أهداف البحث. .

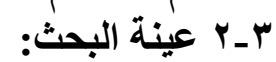

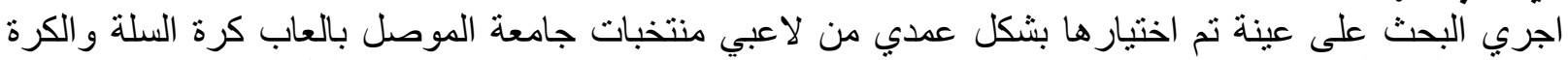

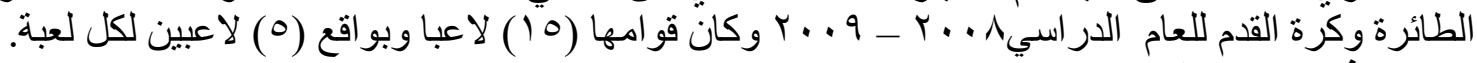

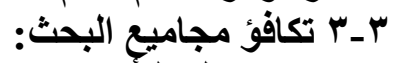

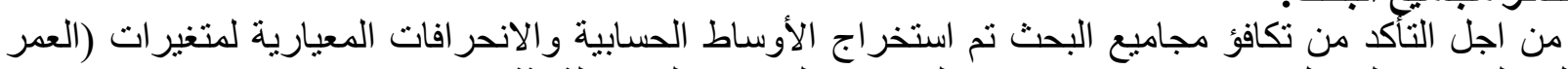

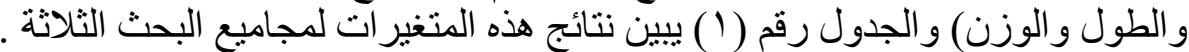

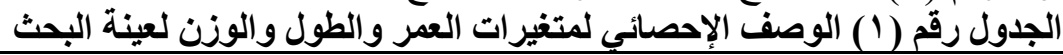

\begin{tabular}{|c|c|c|c|c|c|c|}
\hline \multicolumn{2}{|c|}{ لاعبي كرة القدم } & \multicolumn{2}{|c|}{ لاعبي الكرة الطائرة } & \multicolumn{2}{|c|}{ لاعبي كرة السلة } & \multirow{2}{*}{ المتغيرات } \\
\hline$\varepsilon^{ \pm}$ & س- & $\varepsilon^{ \pm}$ & س- & $\varepsilon^{ \pm}$ & س- & \\
\hline 1 & r. & $1 . r$. & 19.1. & $1.1 \leqslant$ & $r \cdot .7$. & العمر/ سنة \\
\hline r.97 & $17 \Lambda_{6} \Lambda$. & V.97 & IVV & 0.71 & $1 \wedge \varepsilon_{6} \varepsilon$. & الطول/ سم \\
\hline $0, V \wedge$ & $V \varepsilon$ & $\varepsilon_{6} \cdot Y$ & VY,Y. & $7,4$. & $V \theta_{6} Y$. & الوزن/ كغم \\
\hline
\end{tabular}

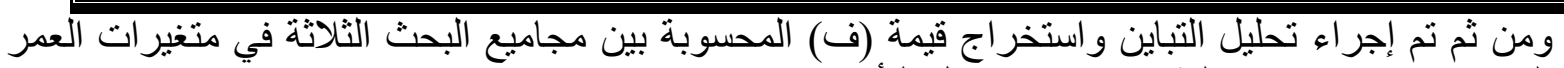

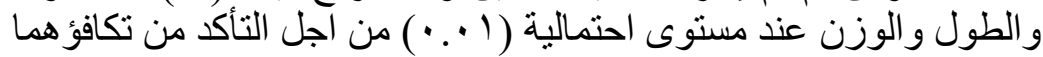
الجدول رقم (r) تحليل التباين في متغيرات العمر والطول والوزن بن بين مجاميع البحث وقيمة (ف) المحسوبة والاحتمالية

\begin{tabular}{|c|c|c|c|c|c|c|}
\hline الاحتمالية & الميمة فية & المربعات & المربعات & الدرجية & مصدر التباين & المتغير ات \\
\hline .049 & .70 &.$\wedge T V$ & 1.VTr & r & بين المجمو عات & العمر \\
\hline
\end{tabular}


اثر جمد لاهواني متكرر في موثر التعب للاعبي كرة السلة..

\begin{tabular}{|c|c|c|c|c|c|c|}
\hline & & אחז.1 & 17 & ir & داخل المجمو عات & \\
\hline & & & IV.VT & $1 \varepsilon$ & المجموع الكلي & \\
\hline \multirow{3}{*}{ חד } & \multirow{3}{*}{ T.01. } & $1 T \cdot . \leqslant 7 V$ & rra. & $r$ & بين المجمو عات & \multirow{3}{*}{ الطول } \\
\hline & & rV. ITV & $\leqslant \leqslant 7$ & IT & داخل المحمو عات & \\
\hline & & & $V .7 .9 \mathrm{VV}$ & $1 \varepsilon$ & المجمو ع الكلى & \\
\hline \multirow{3}{*}{.79.} & \multirow{3}{*}{ • } & $11 . \varepsilon \cdots$ & rY.A.. & $r$ & بين المجمو عاتّ & \multirow{3}{*}{ الوزن } \\
\hline & & rq.A.. & rov.7.. & ir & داخل المجمو عات & \\
\hline & & & $\Gamma \wedge \cdot . \Sigma \cdot$ & Iร & المجموع الكلي & \\
\hline
\end{tabular}

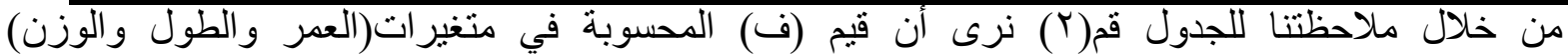

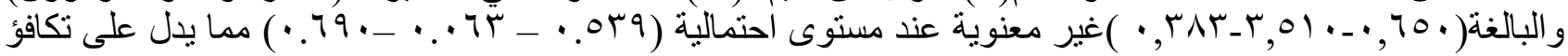

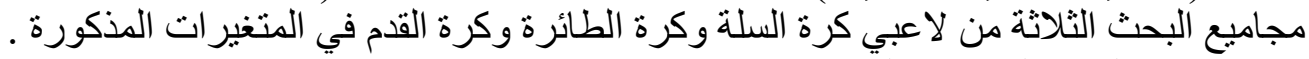

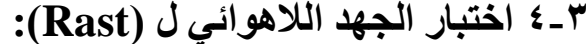

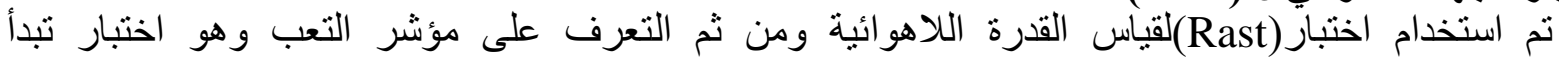

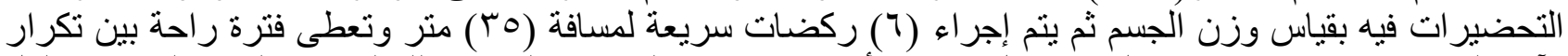

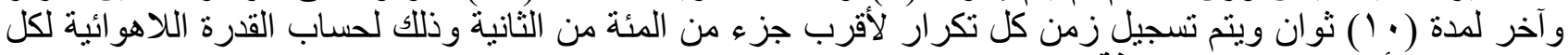

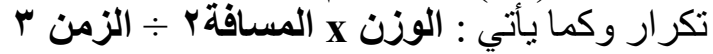

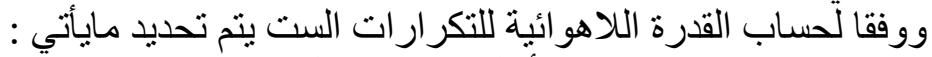

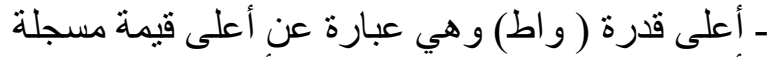

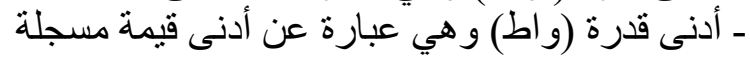

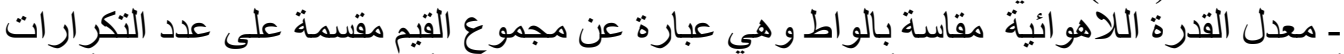

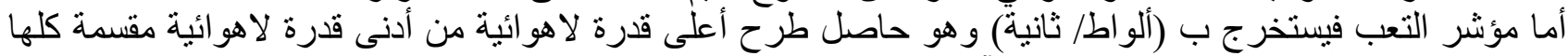

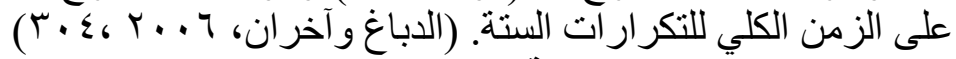

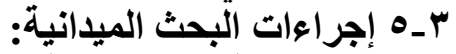

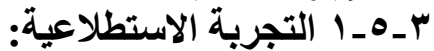

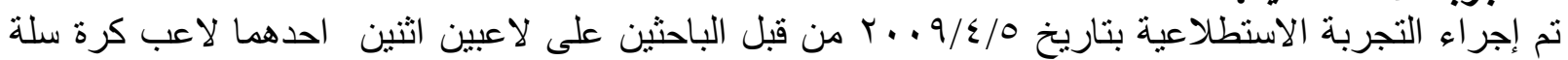

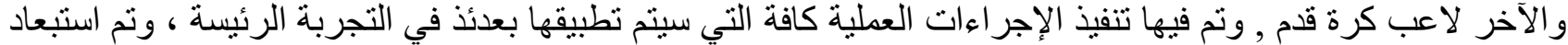

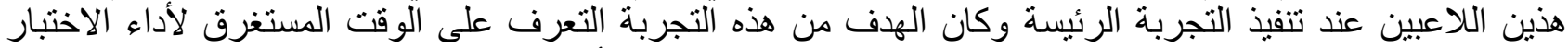

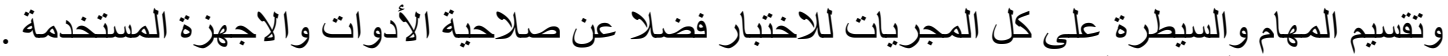

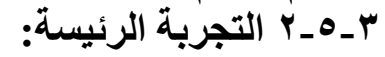

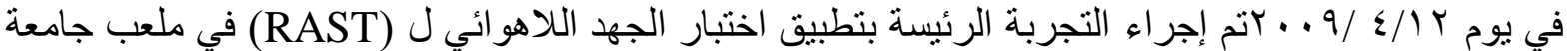

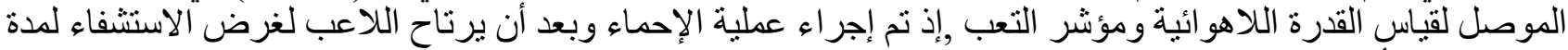

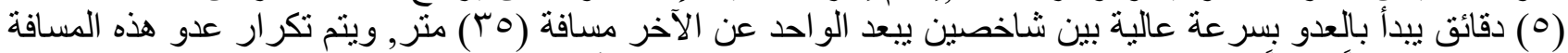

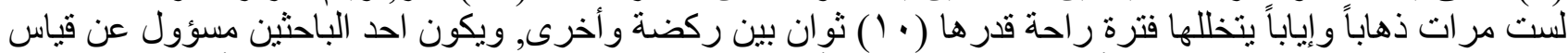

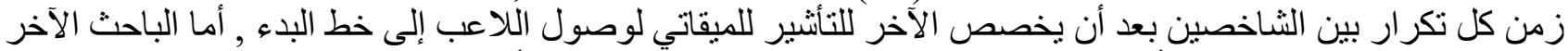

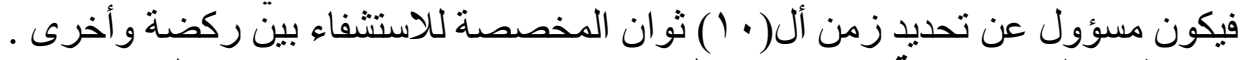

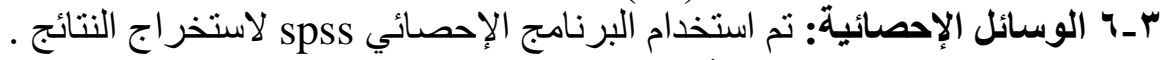

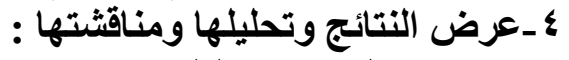

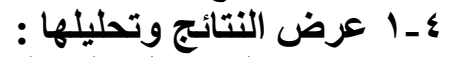

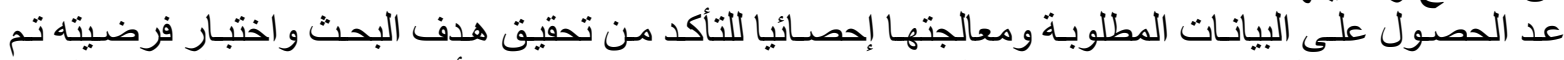

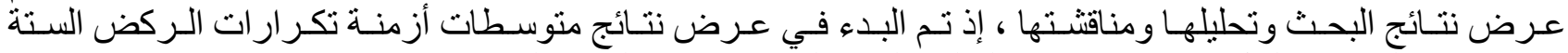

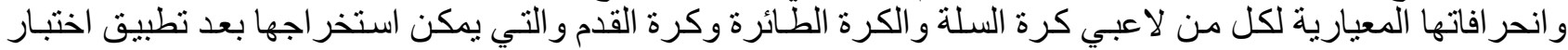
(RAST)

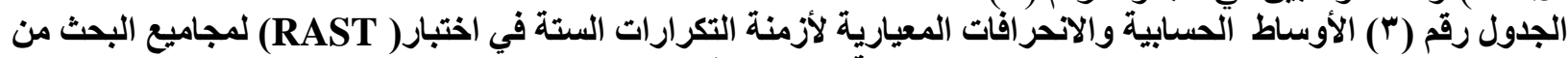
لاعبي كرة السلة والكرة الطائرة وكرة القام

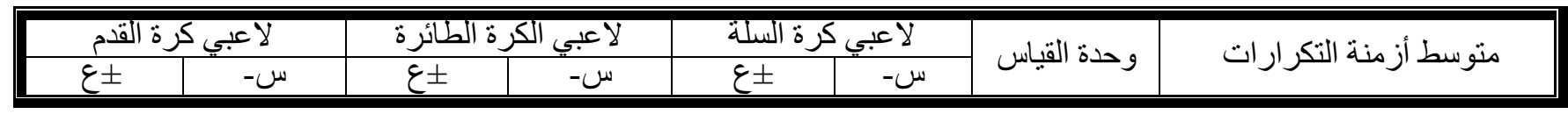


اثر جمد لاهواني متكرر في موثر التعب للاعبي كرة السلة.

\begin{tabular}{|c|c|c|c|c|c|c|c|}
\hline.$Y 7$ & $\varepsilon .0$ & .10 & $\varepsilon . \leqslant \Lambda$ & . ו & $\varepsilon .0$ & تثانية & متوسطزمن التكر ار الاول \\
\hline. $.1 \mathrm{~V}$ & $\varepsilon .01$ &.$Y T$ & $\varepsilon .7 \varepsilon$ &.$\varepsilon \cdot$ & $\varepsilon .7 V$ & ثانية & متوسط زمن التكرار التاني \\
\hline. r人 & $\varepsilon .97$ & .10 & $0.1 Y$ &.$\leqslant \Lambda$ & $0.1 T$ & ثنانية & متوسطزمن التكر ار الثالث \\
\hline$\cdot r q$ & $0.1 \mathrm{~V}$ &.$T V$ & $0 . \leqslant 9$ &.$T V$ & $0 . T Y$ & ثانية & متوسطزمن التكرار الرابع \\
\hline$\because \leqslant 7$ & 0.0. & .19 & 0.71 &.$r \Lambda$ & $0, \pi$. & ثانية & متوسطزمن التكرار الخامس \\
\hline.$\leqslant 1$ & $0,0$. &.$\Gamma \varepsilon$ & 0.17 & . ו & $0 . \leqslant V$ & ثنانية & متوسطزمن التكر ار السادس \\
\hline
\end{tabular}

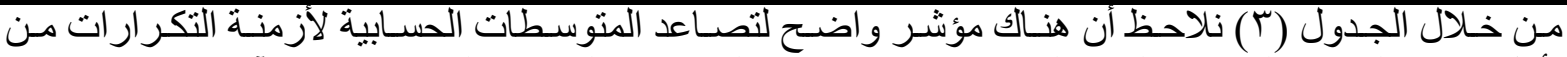

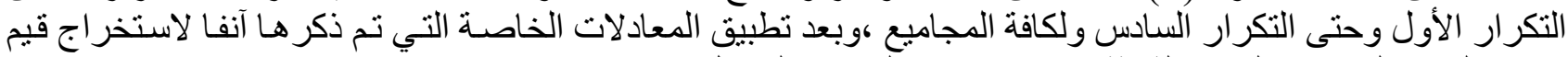

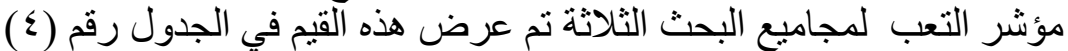
الجدول رقم (؛ ) الأوساط الحسابية والانحر افات المعيارية لمتوسط مؤشر التعب لمجاميع البحث الثلاث من لاعبي كرة السلة

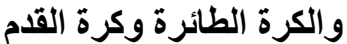

\begin{tabular}{|c|c|c|c|c|c|c|c|}
\hline القدم & لاعبي & الطائرة & لاعبي الَّ & ة السلة & لاعبي & حدة & تخن \\
\hline $\begin{array}{l}\varepsilon^{ \pm} \\
1 . \wedge \wedge 7\end{array}$ & r س. & $\begin{array}{l}\varepsilon^{ \pm} \\
1.0 \leqslant r\end{array}$ & צץא. ו- & $\begin{array}{l}\varepsilon^{ \pm} \\
r .1 \wedge \wedge\end{array}$ & سـ & و اطلثانية & مؤشر التعب \\
\hline
\end{tabular}

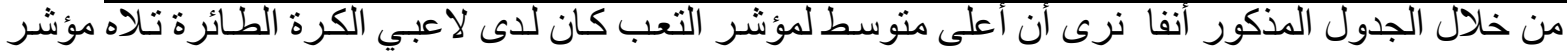

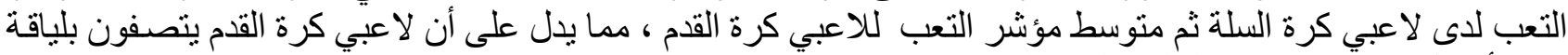

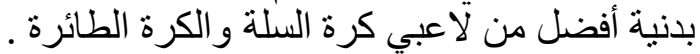

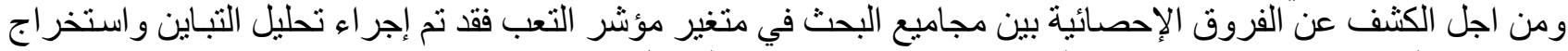

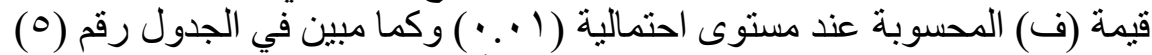
الجدول رقم (0) تحليل التباين في متغير مؤشر آلتعب وقيمتي (ف) المحسوية والاحتمالية

\begin{tabular}{|c|c|c|c|c|c|c|}
\hline الاحتمالية & القيمسة (ف) & المربعات & المربعوع & الحرية & مصدر التباين & المتغير \\
\hline \multirow{3}{*}{$\because \vee \backslash \wedge$} & \multirow{3}{*}{$\cdot r I \leq$} & 1.111 & T.ETV & $T$ & بين المجمو عات & \multirow{3}{*}{ مؤشر } \\
\hline & & r.0Vo & $E Y .9 \cdot T$ & Tr & داخل المجمو عات & \\
\hline & & & 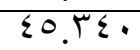 & $1 \varepsilon$ & المجمو ع الكلي & \\
\hline
\end{tabular}

نلاحظ من الجدول رقم (0) عدم وجود فروق ذات دلالة معنوية في مؤشـر التعب ، إذ كانت قيمة (ف) المحسوبة

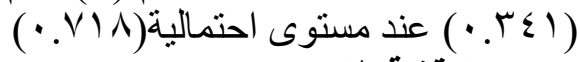

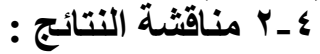

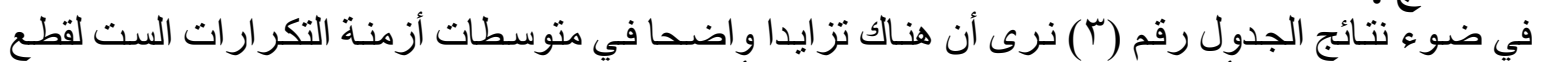

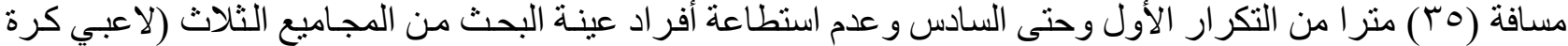

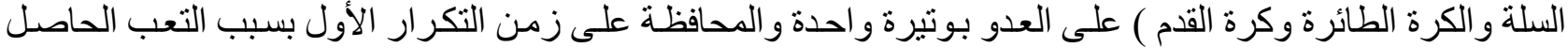

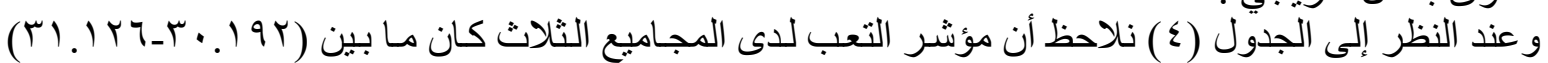

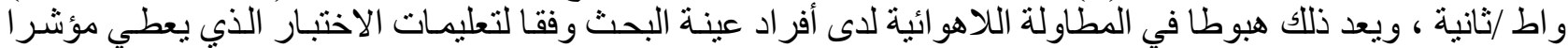
على هذا الهبوط عند تجاوز القيمة (• (1) و اط /ثانية, إذ كلما قل رقم مؤشر التعب كان دليلا على تحسن المستوى آلبدني .

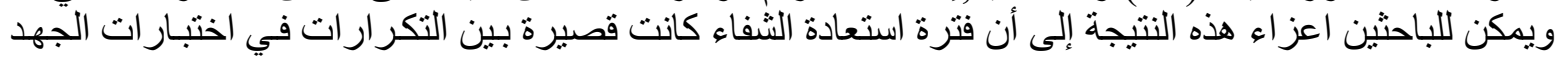

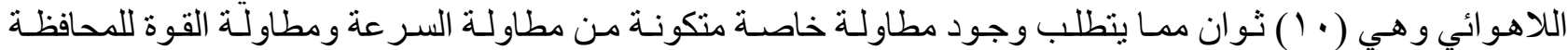

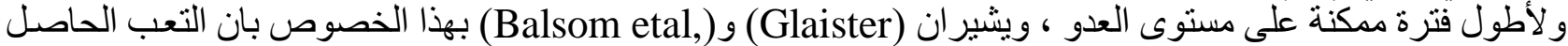
في أثناء الركضات السريعة يظهر على شكل شكل انخفاض متدرج في نتائج القدرة اللاهو ائية التي تعتمد بشكل كبير على فترة

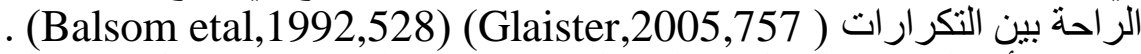
أما الصورة الداخلية ( الفسيولوجية ) لحالة التعب فيعزو ها الباحثون إلى ما يحدث على الفي مستوى البيئة الداخلية

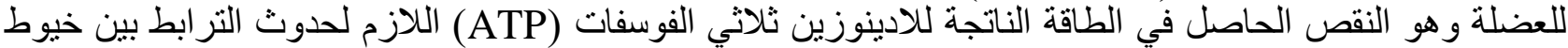

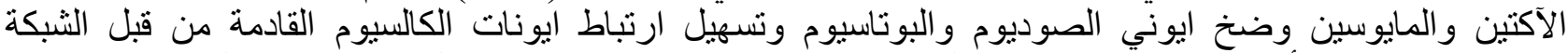
الساركوبلازمية ،إذ أن التعب يحدث نتيجة فثل العمليات الايضية في إعادة تصنيع ال (ATP) بمعدل مناسب لتحقيق 


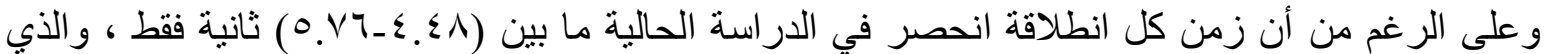

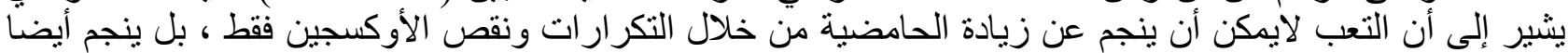
عن تر اكم الفوسفات اللاعضوي (pi ) داخل الخلية العضلية (Walter eta,1997,525) .

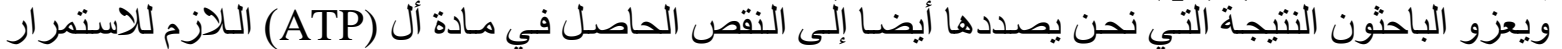

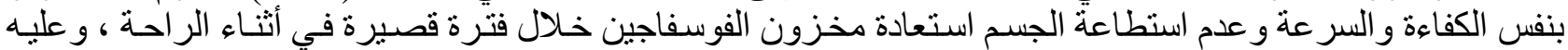

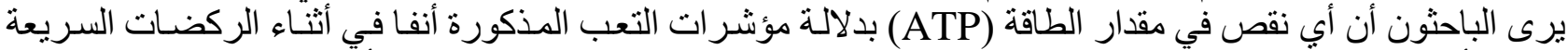

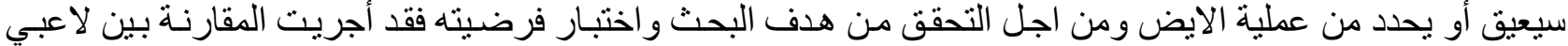
كرة السلة وكرة القدم والكرة الطائرة في مؤشر التعب واظهر الجدول (0) عدم وجود فروق معنوية بين المجاميع التثلاث

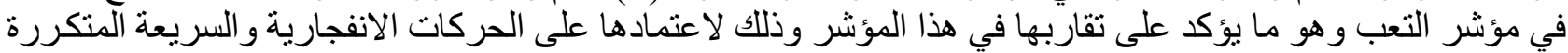

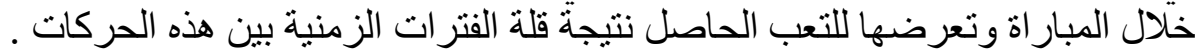

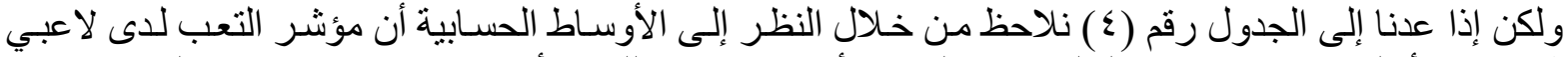

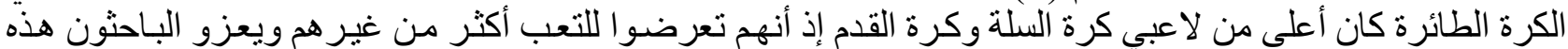

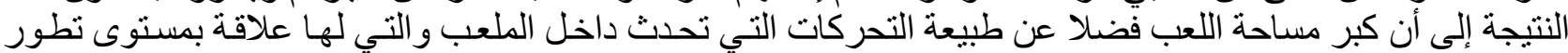

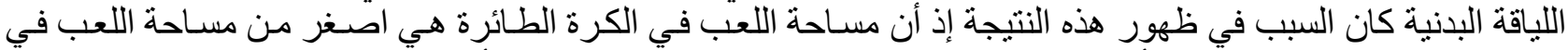

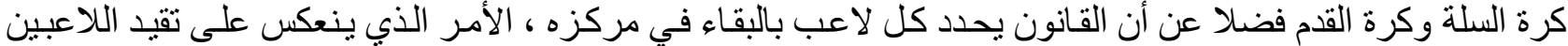

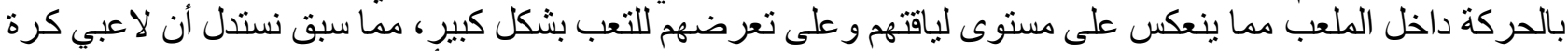

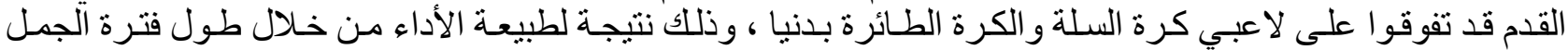

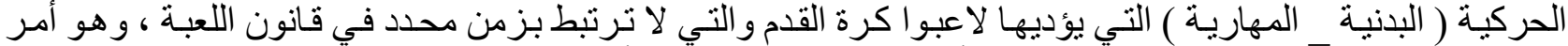

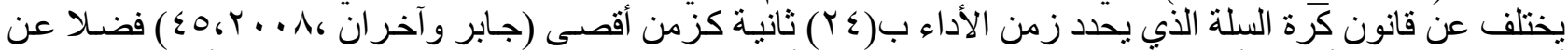

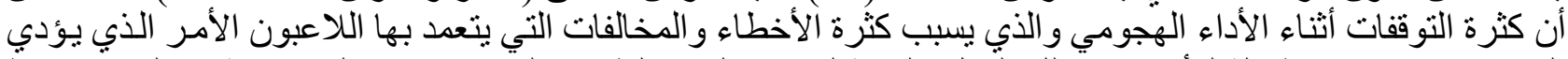

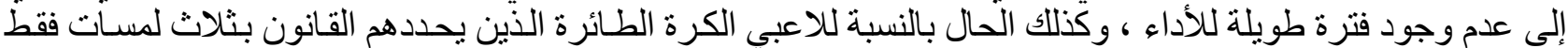

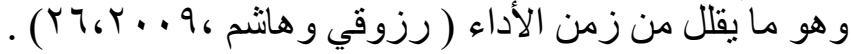

\section{0ـ الاستتناجات والتوصيات:

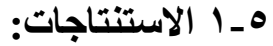

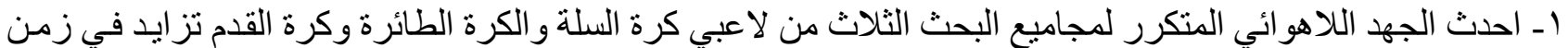

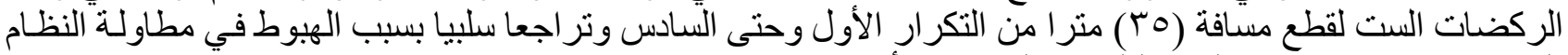

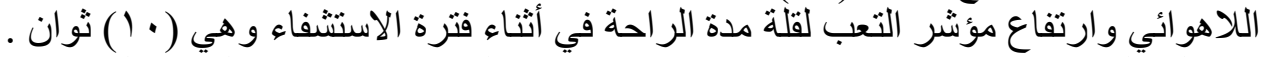

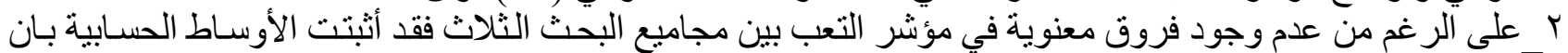

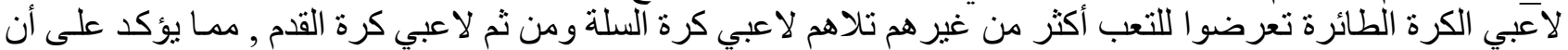

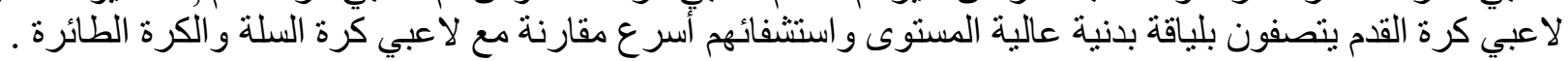
: r_ التوصيات

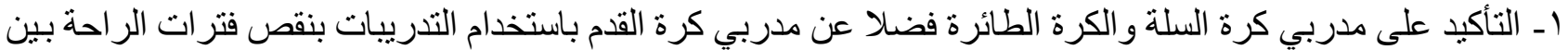

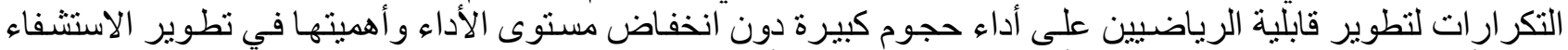

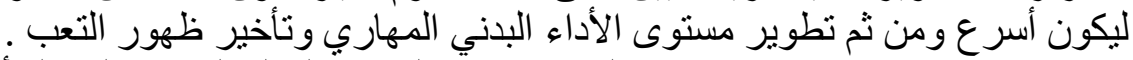

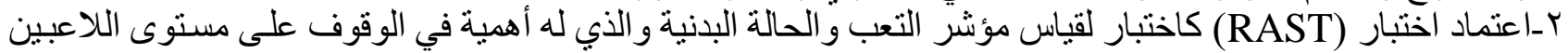
في الفعاليات الرياضية

المصادر العربية والأجنبية:

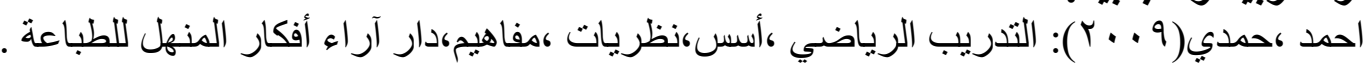

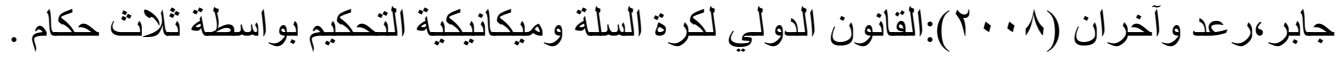

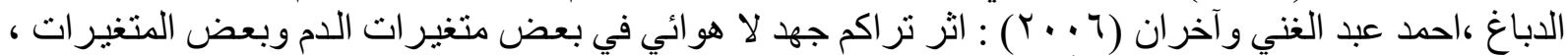

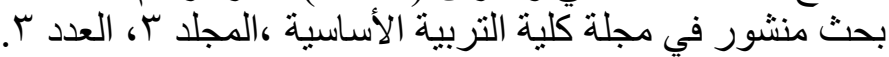

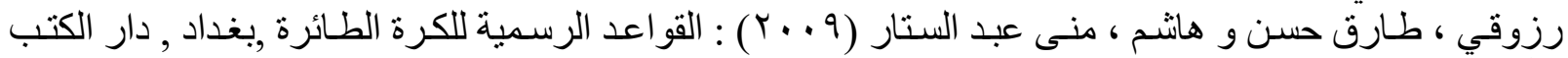
و الوثائقي. 


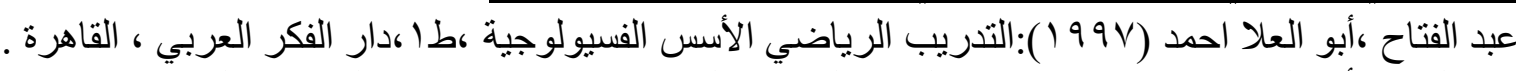

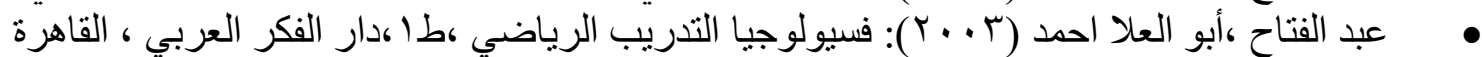

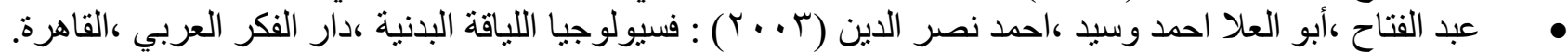

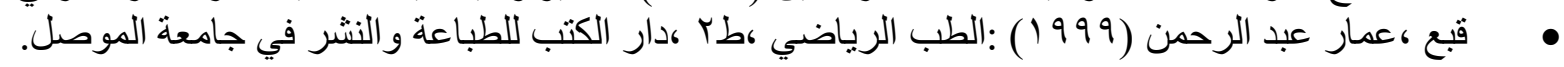

- Balsom PD Seger Jy,sgoin B (1992):Maximal-intensity intermitent exeruse eefeect of recovery duration.

- Glaister M (2005) : Multiple sprint work physiological responces . mechanisms of fatigueand the influence of aerobic fitness .

- Walter , Vandenborn k,Mccully KK(1997): Noninrasire measurement of phosphocrreatine recovery kinetics in single human musclcs . 